Canadian Oncology

Nursing Journal

Revue canadienne

de soins infirmiers

en oncologie

Volume 30, Issue 3 • Summer 2020

elSSN: 2368-8076 


\title{
Facing forward: The development of a cancer nursing knowledge and practice framework
}

\author{
by Allyson Nowell, Colleen Campbell
}

\begin{abstract}
The Canadian Association of Nurses in Oncology (CANO/ACIO) is the national organization supporting nurses to develop and promote excellence in oncology nursing practice, education, research and leadership. To support their mission, CANO/ACIO has developed Standards of Care for cancer patients and Standards and Competencies for Oncology Nurses caring for these individuals (CANO, 2001, 2006). Since the creation of the first standards for specialized oncology nursing in 2006, cancer care has changed considerably with increased cancer occurrence and prevalence, new therapies including oral agents, and cancer care transitioning from specialized treatment centres (Canadian Cancer Society, 2019). Given the changing landscape for nursing practice, CANO/ ACIO embarked on a process to update the current standards with the aim of including the role of nurses caring for cancer patients and families in all settings. Through this process experts identified the need for a national nursing framework to assist with the integration of current standards and describe nursing contributions to high quality cancer care. This article describes the process that CANO/ACIO utilized to establish the CANO Nursing Knowledge and Practice Framework and Toolkit for Cancer Care.
\end{abstract}

\section{BACKGROUND}

$\mathbf{I}_{\mathrm{c}}^{\mathrm{n}}$ $\mathrm{n}$ the context of the increasing complexity of the cancer control system both nationally and internationally, it has become apparent that individuals diagnosed with cancer and their families have myriad needs (Bilodeau et al., 2019; Saria et al., 2017). Whether or not these needs are met, varies across Canada (Shakeel et al., 2020). Considering nearly one in two Canadians may be diagnosed with cancer at some point in their lifetime, and three-quarters of those will live with cancer, nurses in many settings will need to care for patients living with cancer.

Currently, there is a lack of cancer-related content in both undergraduate and graduate nursing education programs. In light of this reality, nurses working within cancer treatment

\section{AUTHOR NOTE}

Allyson Nowell RN, MSc, CON(C)*

Director of Clinical Services

Kensington Hospice

38 Major Street, Toronto, ON M5S 2L1

anowell@kensingtonhealth.org

Colleen Campbell RN-EC, MN, CON(C)

Nurse Practitioner Hematology, Central Region Nursing Lead

Southlake Regional Cancer Centre

Newmarket, ON L3Y 2P9

*Author for contact

DOI:10.5737/23688076303208211 centers have supplemented their specialty learning with online courses, formal and informal mentorship programs, and education or support from their national oncology organization, CANO/ACIO (Charalambouse \& Kaite, 2013; International Society of Nurses in Cancer Care, 2013). However, graduate nurses and nurses practising in non-oncology settings may be lacking essential knowledge and skills in caring for cancer patients and families (Canadian Nurses Association, 2019). Nurses working outside cancer treatment centres have little access to, or encouragement to obtain, additional training in cancer nursing.

Given the intricacy of cancer treatments, the increased use of oral targeted agents, the majority of treatments given in the outpatient setting, and the multidimensional impact of cancer across the cancer trajectory, it is imperative to develop competencies for nurses in all healthcare settings, while advocating for nursing curricula to include the complexity of cancer nursing at the college, undergraduate and graduate levels (Saria et al., 2017; Wood, 2011, Bonnacchi et al.; 2016, Barsky-Reese et al., 2014; Harrington et al., 2010; Cooley, 2000; Soothill et al., 2002).

As the national organization for oncology nurses, in 2001 CANO/ACIO developed Standards of Care focused on patients. Subsequently, in 2006, CANO/ACIO developed Practice Standards and Competencies for the Specialized Oncology Nurse. The emphasis of these standards and competencies was primarily on specialized oncology nursing practice. Additional Standards have been developed, which focus on nursing practice related to care of patients receiving specific modalities of care (i.e., chemotherapy, radiation). All support the provision of high-quality nursing within specialized settings for the diagnosis and treatment of cancer (CANO 2015, 2017). As the delivery of cancer care has shifted from specialized environments, and emphasis has increased on both prevention and survivorship phases of the cancer continuum, national discussions have emerged identifying the need to develop support and guidance for nurses who are involved in cancer related care across all settings.

With continued fiscal restraints and the mounting human resource shortage, it is imperative to have nurses in all regulatory groups working to their full scope of practice (CNA, 2015; Cancer Care Ontario [CCO], 2018). Nurses, educators and professional nursing organization leaders have identified gaps concerning the lack of clarity in standards, competencies, and educational preparation for nurses working in settings other than specialist cancer centres. CANO/ACIO, as the national representative for those nurses, developed a framework as guidance for all nursing roles in all practice settings to provide high quality services that meet the needs of people at risk for and living with cancer. Ultimately, but working collectively as a nursing community, the burden of cancer in Canada could be reduced. 


\section{FRAMEWORK DEVELOPMENT}

\section{Stakeholder \& Expert Engagement}

Within its 2001 Standards of Care document, CANO/ACIO described three categories for oncology nursing roles delineated as generalist, specialist and advanced. The specialist and advanced role categories were well described. With the changes in cancer care and the shifting into ambulatory and community-based delivery, there has been a call to further articulate the generalist role or area of nursing practice in cancer care.

In 2015, CANO/ACIO reached out to members and expert oncology nurses to gather input on the relevance of the Standards of Care, current issues, and gaps in cancer care within Canada using phone interviews $(n=19)$ and holding focus group discussions $(n=42)$. Although there was consensus that the Standards of Care and the Specialized Oncology Nurse Standards and Competencies were still relevant and being used in specialized cancer settings, the nurses pointed to gaps in the other practice settings regarding the nursing care of patients at risk for cancer or living as survivors following the completion of cancer treatment. Through discussions with CANO/ACIO members, the project team identified a need for supporting nurses caring for cancer patients in practice settings outside of the specialist oncology and regional cancer care settings. The need was also identified to integrate the broader family of nursing roles, including Licensed Practical Nurses (LPN) and Registered Practical Nurses (RPN in Ontario) and Advanced Practice Nurses (including Nurse Practitioners and Clinical Nurse Specialists), in the updated documents.

Following this broad input, the CANO/ACIO Project team undertook an international review of work by other oncology nursing organizations regarding oncology nursing roles, standards and competencies. We discovered there had been limited work describing oncology practice beyond that of the Specialist Oncology nurse. One framework from Cancer Australia described four categories of nursing, as part of a professional development model for nursing in cancer care (Aranda \& Yates, 2009). Although the model was designed to support professional development, the framework provided a foundation to describe nursing's contributions in cancer care.

\section{Adapting the Framework}

The CANO/ACIO Board of Directors, led by the Directorat-Large for Professional Practice, (author AN), reviewed Australia's national professional development framework (EdCaN). They discussed how its components could be modified to serve as a nursing care and knowledge framework in which the existing CANO/ACIO Standards would fall (Aranda $\&$ Yates, 2009). The Board members thought adopting and adapting the Australian EdCaN framework would allow for further development of the generalist nursing role in cancer care. Part of the challenge in developing the generalist role was the need to cover a diverse range of settings and care needs (e.g., surgical inpatient unit with majority oncology cases versus community setting with occasional cancer patients and chemotherapy).
Following the Board's decision to work with the Australian EdCaN framework, CANO/ACIO undertook a member engagement process. It was seen as important to gather expert input regarding the EdCaNs relevance to the Canadian practice context. The EdCaN framework was introduced at a workshop during the $2016 \mathrm{CANO} / \mathrm{ACIO}$ conference. The 33 workshop participants held various oncology nursing roles across Canada. Through interactive group discussion, the participants provided input to develop the overarching scope of the Canadian work to adapt the EdCaN framework and define the range of oncology nursing roles, and the necessary competencies of each nursing role. The input supported the idea that the EdCaN framework would be a useful basis for adaptation to a Canadian context. Participants also highlighted the variations in language and practice within the Australian document that would need to be altered for a national Canadian oncology nursing approach.

As a next step in the process of designing our Canadian framework, an expert working group was established consisting of CANO/ACIO members from across Canada. The initial focus of the group was developing the purpose and objectives of the Canadian framework. The definitions of the different categories of nursing roles were reviewed and debate was held about how these nursing roles would work at different points across the cancer continuum, as well as how they fit within the four domains of the framework. The domains in the framework are based on the volume of cancer patients with which a nurse is working in a specific care setting. The four domains are: 'All', 'Many', 'Some', and 'Few'.

Once the language of the framework and its purpose and definitions had been drafted, this version of the Canadian framework was taken to a workshop at the CANO/ACIO national conference in 2017. The purpose of this step was to seek expert member input regarding the clarity of the document and verification that the new framework would be useful. Thirty participants indicated the framework would be useful and began to provide input on the standards and competencies needed to support the domains within the framework.

Following this session, the new framework was taken to the CANO/ACIO Board of Directors for review and final approval. The Board's input was integrated and a unanimous vote approved the framework for adoption (see Figure 1).

\section{CANO NURSING KNOWLEDGE AND PRACTICE FRAMEWORK FOR CANCER CARE}

This section provides an overview of the elements within the new framework.

\section{Purpose of Framework}

- To provide a framework for nursing practice and knowledge to guide care across the continuum for patients with cancer and their families.

- To describe where nurses can be categorized in a domain of the framework depending on the volume of individuals with cancer being cared for in a particular practice setting: 'All Nurses' represents a setting where only a small portion 


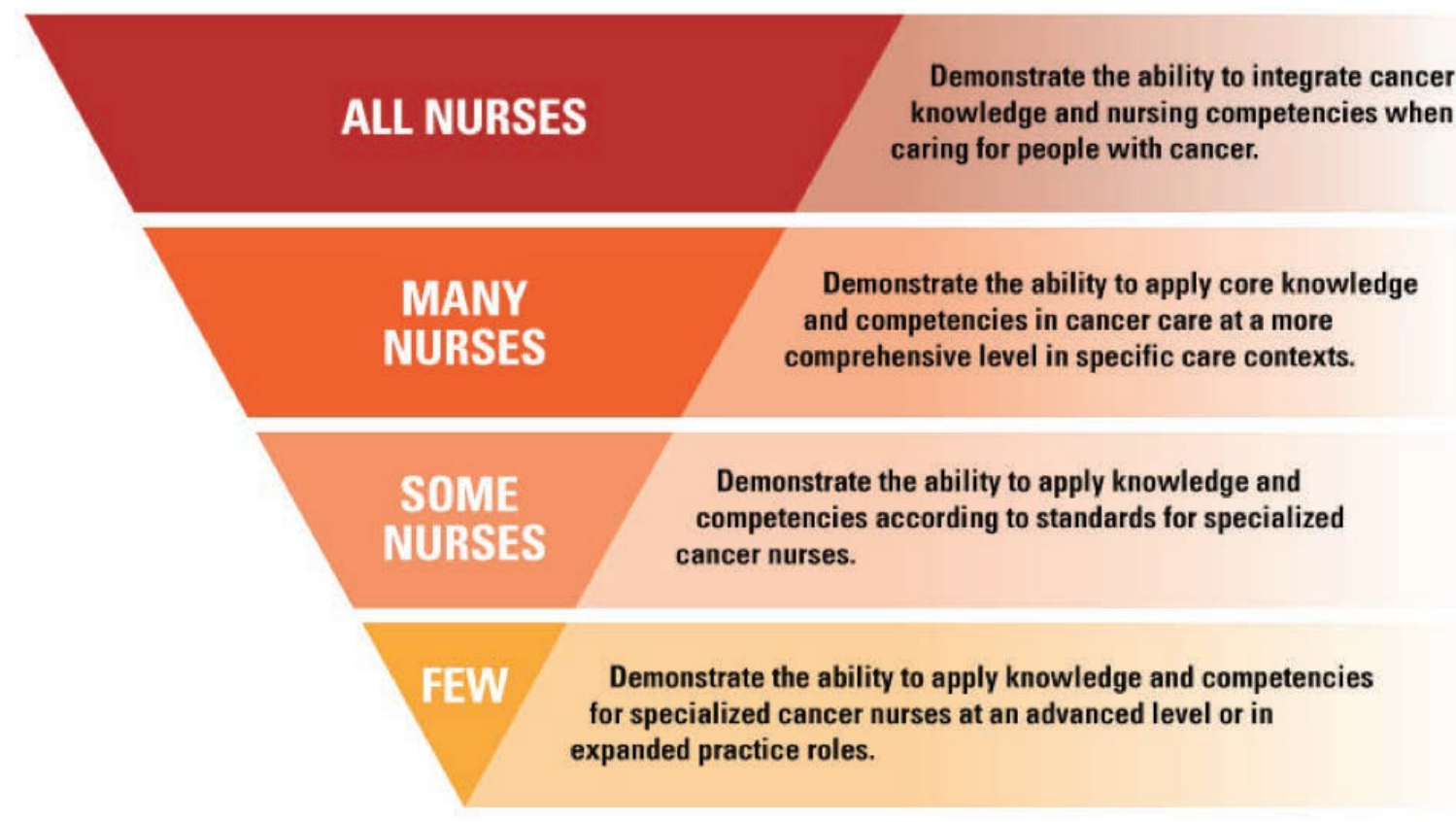

of cancer patients would be part of the care; 'Many Nurses' represents a setting with increasing numbers of cancer patients; 'Some Nurses' reflects a setting where the majority of all patients would have cancer; and 'Few Nurses' represents the practice of an Advanced Practice Nurse within a specialized cancer setting.

Objectives of the Framework

a) highlight nurses' contribution to high quality cancer care

b) describe oncology nursing knowledge required across the continuum of care for cancer patients

c) set out the standards and competencies required for cancer nursing care

d) discuss the various nursing roles across the spectrum of cancer care

Underlying Assumptions of the Framework

- Highlights oncology knowledge expected of nurses irrespective of where they work

- Ensures the best care of cancer patients

- Emphasizes collaboration of all nurses across continuum is essential

\section{CANO NURSING KNOWLEDGE AND COMPETENCIES FRAMEWORK FOR CANCER CARE}

Development of the Framework Toolkit

Following the launch of the CANO/ACIO Nursing Knowledge and Competencies Framework for Cancer Care, the CANO/ACIO Professional Practice Committee identified a need for resources to support the uptake of the framework across care settings. During the 2017 workshop, participants had verified that the 2006 Specialized Oncology Nurse Standards were relevant for all nursing practice and could be adapted to reflect the four domains of the Framework. The Committee began to draft an accompanying document and toolkit that described the need for the Framework across care settings, discussed all nursing roles caring for individuals with cancer, and established high level practice standards. Clinical scenarios were included to build a better understanding of cancer care across all settings.

The process for drafting the final document and toolkit included members of the CANO/ACIO Professional Practice Committee, as experts in oncology nursing, reviewing the existing standards and determining how they would apply them in all domains of the framework to develop competencies. Each standard was completed by pairs, then reviewed and refined with the whole group. In order to be assured that the completed document was relevant and applicable in non-specialized oncology settings, a stakeholder survey was created. This was circulated to nurses working in non-oncology specific settings. Eighteen responses were received and an overwhelming majority $(94 \%)$ of these respondents agreed the document was applicable to their setting. The document was seen as demonstrating the depth of nursing knowledge related to cancer care required for nurses in non-specialized oncology settings.

The final document, entitled The Nursing Knowledge and Practice Framework for Cancer Care, highlights oncology knowledge expected of all nurses irrespective of where they work and is meant for use by nurses, leaders, educators, and decision makers.

\section{Summary}

Foundational oncology knowledge is required for all nurses working with cancer patients and families regardless of setting or license. Given the growing population living with cancer and the number of treatment modalities given as an outpatient, every nurse may care for cancer patients and families regardless of the setting in which they work, including long-term care, primary care and hospital inpatient units. CANO/ACIO developed Practice Standards and Competencies for the Specialized Oncology Nurse (2006) recognizing the gaps in education and the need to support high quality oncology nursing. However, 
many nurses outside treatment facilities are unaware of the standards, or how to adapt them in the non-specialized oncology setting. Recognizing the need to support these nurses, CANO/ ACIO adopted the Nursing Knowledge Framework for Cancer Care. To support knowledge translation of the Framework, a toolkit outlining how the standards and expected competencies fit within the framework was developed.

\section{CONCLUSION}

Given the increasing number of cancer patients and survivors within our Canadian population, nurses practising in any care setting are likely to encounter patients and families at various points along the continuum of the cancer experience. Nurses in specialized treatment settings have had support and resources to grow and continue to hone their competency, however the vast majority of nurses working in hospitals or community have lacked the education or practice standards around cancer care required to support their professional practice in

\section{REFERENCES}

Aranda, S., \& Yates, P. (2009). A national professional development framework for cancer nursing. (2nd ed.). The National Cancer Nursing Education Project (EdCaN). https://www.Edcan.org.au/ professional-development/edcan-framework

Barsky Reese, J., Porter, L., Regan, K., Keefe, F., Azad, N., Diaz, L., Herman, J., \& Haythornthwaite, J. (2014). A randomized pilot trial of a telephone-based couples intervention for physical intimacy and sexual concerns in colorectal cancer. Psycho-oncology, 23(9), 1005-1013. https://doi.org/10.1002/pon.3508

Bilodeau, K., Tremblay, D. \& Durand, M.J. (2019). Gaps and delays in survivorship care in the return-to-work pathway for survivors of breast cancer-A qualitative study. Current Oncology, 26(3), http:// dx.doi.org/10.3747/co.26.4787

Bonacchi, A., Miccinesi, G., Galli, S., Primi, C., Chiesi, F., Lippi, D., Muraca, M., \& Toccafondi, A. (2016). Use of the Needs Evaluation Questionnaire with cancer outpatients. Supportive Care in Cancer, 24(8), 3507-3515.

Canadian Association of Nurses in Oncology. (2006). Practice Standards and Competencies for the Specialized Oncology Nurse. https://cdn.ymaws.com/www.cano-acio.ca/resource/resmgr/ standards/CONEP_Standards2006September.pdf

Canadian Association of Nurses in Oncology (2001). Standards of Care. https://cdn.ymaws.com/www.cano-acio.ca/resource/resmgr/ standards/Standards_of_Care_ENG.pdf

Canadian Association of Nurses in Oncology (2017). Standards and Competencies for Cancer Chemotherapy Nursing Practice. https://cdn. ymaws.com/www.cano-acio.ca/resource/resmgr/Resources/EN_ CANO_Chemotherapy_Standar.pdf

Canadian Association of Nurses in Oncology (2015). Radiation Oncology Nursing Practice Standards and Competencies. https:// cdn.ymaws.com/www.cano-acio.ca/resource/resmgr/standards/ ronp_s\&c_web(2).pdf

Canadian Cancer Society (2019). Cancer Statistics at a glance. http://www.cancer.ca/en/cancer-information/cancer-101/ cancer-statistics-at-a-glance/?region $=$ on

Canadian Nurses Association. (2015). Framework for the Practice of Registered Nurses in Canada. https://www.cna-aiic.ca/-/media/cna/ page-content/pdf-en/framework-for-the-pracice-of-registerednurses-in-canada.pdf caring for individuals with cancer. The CANO/ACIO Nursing Knowledge and Practice Framework in Cancer Care describes nurses' contribution to high quality cancer care, acknowledges the nursing knowledge required to provide that care, and supports all nursing roles across the continuum of cancer care. A toolkit to support knowledge translation of the Framework has been developed and integrates standards for all four domains outlined in the Framework. The goal of this resource is to provide a helpful foundation to support the preparation of nurses and their practice in caring for cancer patients irrespective of their practice setting or educational preparation.

\section{ACKNOWLEDGEMENTS}

The authors would like to acknowledge the entire Professional Practice Committee for their work in developing the Framework Toolkit and all the oncology nursing experts who provided input throughout its development.

Canadian Nurses Association. (2019). Advanced Practice Nursing, A Pan-Canadian Framework. Canadian Nurses Association: $\quad$ https://cna-aiic.ca/-/media/cna/page-content/ pdf-en/apn-a-pan-canadian-framework.pdf?la=en\&hash= E1387634D492FD2B003964E3CD4188971305469E

Cancer Care Ontario: Ontario Oncology Advanced Practice Nursing Community of Practice (2018). Expert recommendations report on clinical nurse specialist and nurse practitioner roles in the delivery of adult cancer services in Ontario. https://www.cancercareontario.ca/ en/guidelines-advice/types-of-cancer/52201

Charalambouse, A., \& Kaite, C., (2013). Undergraduate nursing students caring for cancer patients: hermeneutic phenomenological insights of their experiences. BMC Health Services, 13(63). https://doi.org/10.1186/1472-6963-13-63

Cooley, M. (2000). Symptoms in adults with lung cancer. Journal of Pain and Symptom Management, 19(2), 137-153.

Harrington, C. B. C., Hansen, J. A., Moskowitz, M., \& Todd B. L. (2010). It's not over when it's over: Long-term symptoms in cancer survivors: A systematic review. International Journal of Psychiatry in Medicine, 40(2), 163-181.

International Society of Cancer Nursing. (2013). Cancer Nursing Education Position Statement. https://cdn.ymaws.com/www.isncc. org/resource/resmgr/Position_Statements/ISNCC_Cancer_ Nursing_Educati.pdf

Saria, M., Nyamathi, A., Phillips, L., Stanton, A., Evangelista, L., Kesari, S., \& Maliski, S. (2017). The hidden morbidity of cancer: Burden in caregivers of patients with brain metastases. The Nursing Clinics of North America, 52(1), 159-178.

Shakeel, S., Tung, J., Rahal, R., Finley, C. (2020). Evaluation of Factors Associated with Unmet Needs in Adult Cancer Survivors in Canada. JAMA Netw Open, 3(3), e200506. https://doi.org/10.1001/ jamanetworkopen.2020.0506

Soothill, K., Morris, S. M., Harman, J. C., Francis, B., Thomas, C., \& Mcillmurray, M. B. (2002). Informal carers of cancer patients: What are their unmet needs? Health and Social Care, 9(6), 476-489.

Wood, L. (2011). A review on adherence management in patients on oral cancer therapies. European Journal of Oncology Nursing, 16(4), $432-438$. 